\title{
Laser Assisted Disinfection in Regenerative Endodontics with CBCT Follow Up - Case Series
}

\section{Divya Doddamani*, Saraswathi V. Naik, Basappa Nadig, Sanjana Ratnakar Kodical, Swapna Shailendra Virdikar and Payel Banerjee}

Department of Pedodontics and Preventive Dentistry, Bapuji Dental College and Hospital, Davangere, India

*Corresponding Author: Divya Doddamani, Department of Pedodontics and Preventive Dentistry, Bapuji Dental College and Hospital, Davangere, India.
Received: October 25, 2021

Published: November 15, 2021

(C) All rights are reserved by Divya

Doddamani., et al.

\begin{abstract}
Management of necrotic young permanent teeth poses greater endodontic challenge to the clinicians. Regenerative endodontics involves the interplay of three key elements of tissue engineering-stem cells, scaffolds, and growth factors. Along with this, efficient root canal disinfection plays a major role. These procedures rely heavily on root canal disinfection. Traditionally used irrigants and medicaments were reported to show drawbacks. Therefore, the purpose of the report is to present the results of laser assisted disinfection in regenerative endodontic cases. Case series describes about the management of four cases of necrotic young permanent teeth. Cases were treated using laser assisted disinfection method. Blood clot was used as a scaffold and coronal seal was given using Mineral trioxide aggregate and final restoration was placed. Laser assisted disinfection combined with blood clot scaffold systems lead to apical closure and increasing radicular thickness in necrotic young permanent teeth. Laser assisted disinfection has been confirmed as an effective adjunct for root canal disinfection. This is the rarest case series which documents the efficacy of a new disinfection protocol in pulp regeneration which showed quantitative outcome of Regenerative Endodontic Therapy when followed for 12 months with CBCT follow up.
\end{abstract}

Keywords: Regenerative Endodontics; Laser; Disinfection; Sodium hypochlorite; Tooth Apex

\section{Abbreviations}

CBCT: Cone Beam Computerized Tomography; NaOCl: Sodium Hypochlorite; EDTA: Ethylenediaminetetraacetic Acid; MTA: Mineral Trioxide Aggregate

\section{Introduction}

Regenerative endodontics is one of the remarkable advancements which stands as a pre-eminent solution for management of immature permanent teeth with pulpal necrosis when dental practitioners are challenged by the unique morphological features of these teeth showing incomplete root apex and thin dentinal walls [1].
The success of regenerative endodontic treatment depends on the eradication of microbes from the root canal system and the prevention of reinfection [2]. Uncontrolled infection can cause damage to tissue forming cells, in turn hamper repair and regeneration. Histologic study of a human immature permanent premolar with chronic apical abscess after revascularization resulted in the formation of soft connective tissue similar to that in the periodontal ligament and cementum-like or bone-like hard tissue [3]. Stem cells of apical papilla introduced in the canal space showed an osteogenic differentiation instead of odontogenic differentiation in the presence of bacterial by-products [4]. Therefore, the intra-radicular infection should be controlled for pulp tissue regeneration [5]. 
Efficient removal of debris and microorganism can be achieved by various techniques like irrigation, instrumentation and intra canal medicaments [6]. Multiple researches have been published with significant differences in disinfection protocols using various disinfecting solutions, antibiotics in regenerative endodontics [7].

Laser technology applied to endodontics has various methods of disinfection such as Direct laser irradiation, Photodynamic therapy, Laser activated irrigation. Lasers assisted disinfection methods can always be used effectively with other traditional disinfection methods [8]. Primary goal of laser in disinfection is to eradicate microorganisms, removal of debris and smear layer which will ensure successful endodontic therapy. Reduction in the bacterial count up to $74 \%$ was noted when $810 \mathrm{~nm}$ diode laser was used [9]. Efficacy of diode laser has been proved in maturogenesis of young permanent teeth [10].

Recently immature teeth treated with regenerative endodontics where photo activated disinfection has been used as an adjunct with irrigating solutions showed root closure and increasing radicular thickness in necrotic immature teeth when followed for 10 to 12 months $[11,12]$. This case series aims to describe the regenerative endodontic outcome in necrotic young permanent teeth when treated with laser assisted disinfection.

\section{Case Report}

Procedure $[13,14]$

\section{First appointment}

Local anaesthesia with vasoconstrictor was administered (Lignox $2 \% \mathrm{~A}-1: 80,000$ adrenaline), rubber dam application was carried out. Access cavity was prepared using round bur (ISO 012/25 $\mathrm{mm})$.

Copious, gentle irrigation was done with $20 \mathrm{ml}$ of $1.5 \% \mathrm{NaOCl}$ (Sodium Hypochlorite) with side vented closed needle $(20 \mathrm{ml} / \mathrm{ca}-$ nal, $5 \mathrm{~min}$ ), followed by disinfection was performed with $810 \mathrm{~nm}$ diode laser (AMD LASERS Picasso Dental Diode Laser) with power $1 \mathrm{~W}, 20 \mathrm{~ms}$ Pulse length and $20 \mathrm{~ms}$ interval duration. The optical fibre tip (200 micron) introduced $1 \mathrm{~mm}$ short of the working length and laser irradiation was performed with the irrigant in the canal for $15 \mathrm{~s}$ [14] followed by 17\% EDTA (Ethylenediaminetetraacetic acid) irrigation. Access cavity was sealed with restorative material (GC Fuji Glass Ionomer Cement) and patient was recalled after 1-4 weeks.

\section{Second appointment}

Local anaesthesia without vasoconstrictor (Lignox 2\%) was administered. Copious, gentle irrigation with $20 \mathrm{ml}$ of $17 \%$ EDTA. Blod clot scaffold was placed inside the root canal by over instrumenting with pre-curved 15 no K file (MANI Inc., Japan). Collagen plug (Colla Cote (TM)) was placed over the blood clot. 3-4 mm MTA (Mineral trioxide aggregate) (MTA Plus) coronal seal was placed.

CBCT (Cone beam computerized tomography) Evaluation [15]

A CBCT scan (Orthophos SL 3D, Firmware V05.12.06 and $5 \times$ $5.5 \mathrm{~cm}$ field of view; $10 \mathrm{~mA}, 85 \mathrm{kVp}$, Dentsply Sirona, Bensheim, Germany) was obtained for radiographic assessment of pre-operative and follow up periods.

\section{Case 1}

A 11-year-old boy was referred to Department of Paediatric Dentistry for endodontic treatment of an immature necrotic tooth. Patient gave a history of trauma a month ago. Clinical examination revealed normal soft tissues and that tooth 11 was negative to electric pulp test. All probing depths were $3 \mathrm{~mm}$. Percussion testing revealed tenderness wrt 11 . CBCT examination showed an open apex. Periapical radiolucency at the apical $3^{\text {rd }}$ (Figure $1 \mathrm{a}, 1 \mathrm{~b}$ ). A diagnosis of necrotic pulp with symptomatic apical periodontitis was made, and REP (Regenerative Endodontic Procedure) was elected as the treatment of choice. Clinical procedures for REP were performed. At 12 months follow up tooth was clinically asymptomatic, CBCT follow up showed an increase in the dentin thickness in the sagittal section, reduction in the size of the periapical radiolucency at the apical $3^{\text {rd }}$ and evidence of bone formation (Figure 1c, 1d).
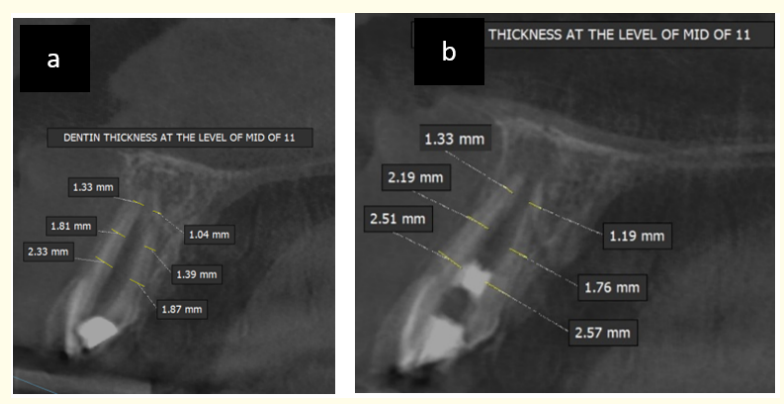

Figure 1: a) Preoperative CBCT wrt 11 (Sagittal Section) shows periapical radiolucency.

b) 12 months follow up CBCT wrt 11(Sagittal Section) shows increase in the dentin thickness. 
Case 2

A 9-year-old boy was referred to Department of Paediatric Dentistry for endodontic treatment of an immature necrotic tooth. Patient gave a history of trauma 2 months ago. Clinical examination revealed normal soft tissues and tooth 21 was negative to electric pulp test. All probing depths were normal. Percussion testing revealed tenderness wrt 21. Radiovisiography and CBCT examination showed an open apex. Periapical radiolucency at the apical $3^{\text {rd }}$
(Figure 2a, 2b, 2c). A diagnosis of necrotic pulp with symptomatic apical periodontitis was made, and REP was elected as the treatment of choice. Clinical procedures for REP were performed. At 12 months follow up tooth was clinically asymptomatic, CBCT follow up showed an increase in the dentin thickness was seen in the sagittal section, reduction in the size of the periapical radiolucency at the apical $3^{\text {rd }}$ and evidence of bone formation (Figure $2 c, 2 d$ ).
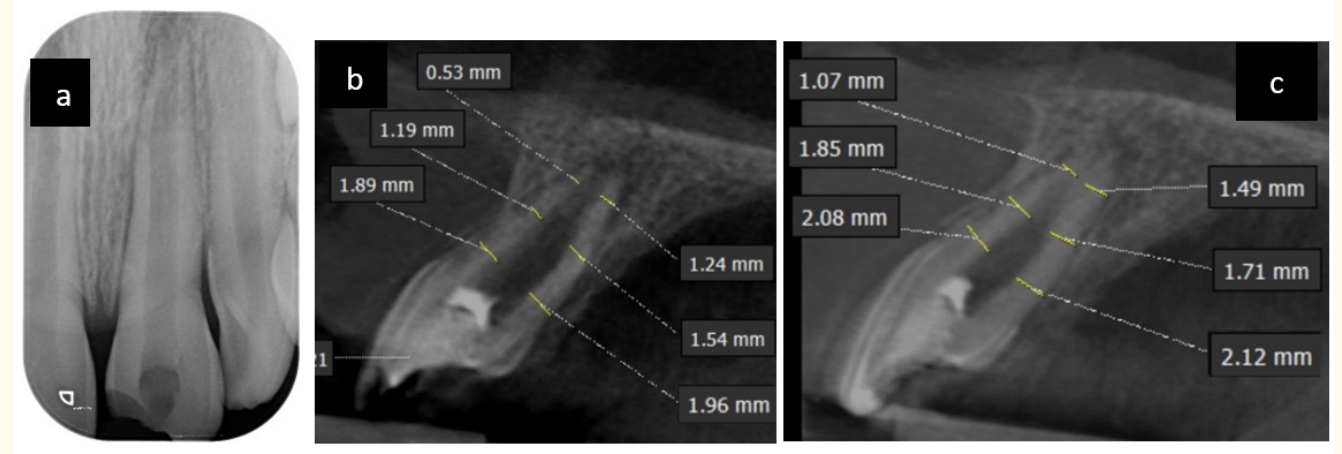

Figure 2: a) Preoperative Radiograph wrt 21 shows periapical radiolucency and open apex.

b) Post-operative CBCT wrt 21 (Sagittal section) periapical radiolucency and open apex.

c) 12 months follow up CBCT wrt 21 (Sagittal section) shows increase in the dentin thickness and blunt end apical closure and reduction in periapical radiolucency.

\section{Case 3}

An 8-year-old boy reported with chief complaint of pain in the lower front teeth region since 3 weeks. Patient gave a history of trauma 2 months ago. Clinical examination revealed normal soft tissues and tooth 31 negative to electric pulp test. Percussion testing revealed tenderness wrt 31. Radiovisography wrt 31 showed an open apex (Figure 3a). CBCT evaluation showed periapical radiolucency and open apex measuring $2.1 \mathrm{~mm}$. Periapical radiolucency at the apical $3^{\text {rd }}$. (Figure $3 \mathrm{~b}$ ) A diagnosis of necrotic pulp with symptomatic apical periodontitis was made, and REP was elected as the treatment of choice. Clinical procedures for REP were performed. At 11 months follow up tooth was clinically asymptomatic, radiographic follow up showed increased root length and apical closure and complete resolution of periapical radiolucency (Figure $3 c)$. Patient could not report for the follow up due to COVID-19 pandemic due to which CBCT follow up was not done.
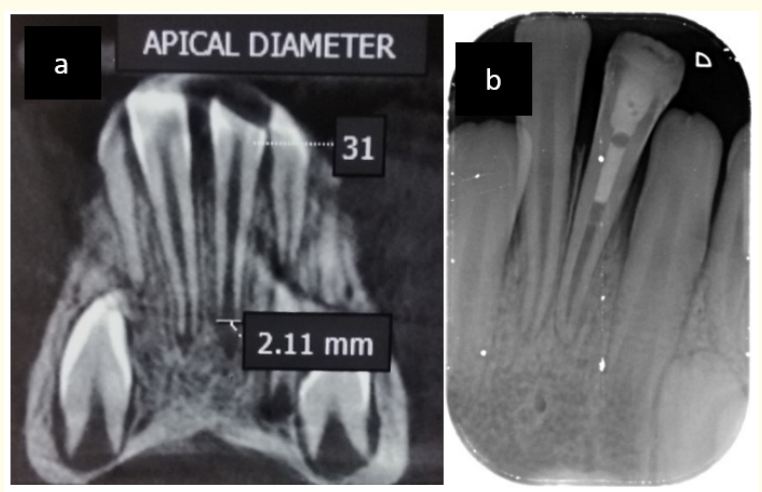

Figure 3: a) Preoperative CBCT wrt 31 (Coronal section) shows periapical radiolucency and open apex.

b) 12 months follow up radiograph wrt 31 shows complete resolution of periapical radiolucency, increase in root length, apical closure. 


\section{Discussion}

Importance of promoting sterile intracanal environment resulting in genetic induction and continuity of root formation was often quoted in literature. In an infected tooth, biofilm formation on the canal walls, contamination of canals by bacterial toxins, and root canal resorption will change the microenvironment around the stem cells. Release of pro-inflammatory cytokines, such as tumour necrosis factor-alpha, interleukin - 1alpha are capable of inhibiting stem cell differentiation [16]. Histobacterial examinations confirmed the inadequate root canal disinfection and inadequate removal of biofilm and bacteria in dentinal tubules [17]. Minimal or no instrumentation of the dentinal walls is always advocated in regenerative endodontic procedures. The disinfection approach was chemical rather than chemo-mechanical. The sterile necrotic pulp and blood clot scaffold are essential in providing a lattice for cell growth [18].

REP using a non-antibiotic disinfectant approach provides satisfactory outcomes for a non-vital immature permanent tooth [19]. Photoactivated disinfection which is already used in the field of regenerative endodontics resulted in successful outcome when followed for 10 to 12 months. Photoactivated disinfection technique was effective in removing high bacterial concentrations from infected root canals. Laser phototherapy was able to influence the proliferation and differentiation of stem cells. Therefore, photoactivated disinfection was effective even in case of single visit Regenerative endodontic cases. These case reports cited in the literature on regenerative endodontics with laser disinfection proves the benefits of laser in successful outcomes such as increase in the dentin thickness, root length and resolution of periapical radiolucency [11,12]. Gallium-Aluminum-Arsenide diode laser irradiation on stem cells from human exfoliated deciduous teeth did not cause a cytotoxic effect [20]. A mean bacterial reduction of 74\% was achieved in root canal wall dentin with the $810 \mathrm{~nm}$ diode laser at a setting of $3 \mathrm{~W}$ in continuous mode for $30 \mathrm{~s}^{9}{ }^{9} 980 \mathrm{~nm}$ diode laser irradiation was effective for disinfecting the root canals containing aerobic and anaerobic bacteria [21]. The laser activation of irrigating solutions such as EDTA or $\mathrm{NaOCl}$ will enhance the removal of the smear layer and bacterial biofilm. After absorption of laser energy by the solution, there will be formation of vapor bubbles, collapse of the bubbles, acoustic streaming, and finally cavitation. Cavitation results in the formation of shockwaves characterized by rapid changes in pressure and high amplitude resulting in disruption of bacterial cell wall and cell death [22,23].

Although antibiotics used in regenerative endodontic procedures are effective against bacteria related to endodontic infections, the main disadvantage of triple antibiotic paste is tooth discoloration caused by minocycline and detrimental effect on stem cells at higher concentration [24]. Triple antibiotic paste has a highly acidic pH (2.9) that affects the microhardness of dentin [25]. Calcium hydroxide has long been used as an intracanal medicament in endodontics. It can disinfect the canal, without any detrimental effect to stem cells. As well as helps in the release of growth factors such as TGF-beta and conducive to SCAP survival and proliferation [26].

CBCT being a quantitative imaging modality, gives a more reliable and accurate details on root growth, periapical pathology compared to periapical radiographs. Limited FOV CBCT imaging has been previously used to evaluate the outcome of REP. CBCT is highly recommended for initial evaluation and follow up visits $[13,15]$.

Blood clot scaffold is the gold standard for regenerative endodontic therapy in younger population because of its extremely favorable and clinically feasible properties among paediatric patients. Blood clot being cell-homing-based approach, the patient's endogenous cells undertake tissue repair/regeneration. Growth factors present in blood clot scaffold plays an important role in cell differentiation. The growth factors such as platelet-derived growth factor, vascular endothelial growth factor, and platelet-derived epithelial growth factor from blood clot promotes regeneration [27].

Present case series shows 3 cases treated with laser assisted disinfection, calcium hydroxide intra canal medicament and blood clot as scaffold. Case 1 and Case 2 showed open apex and periapical radiolucency during CBCT evaluation. 12 months follow up showed considerable reduction in the size of the peri radicular radiolucency and increase in the dentin thickness in sagittal sections. This indicates incomplete healing and these cases have to be followed up further. Case 3 showed an open apex, periapical radiolucency. At 12 months follow up there was complete resolution of periapical radiolucency, increase in root length, apical closure indicating complete healing. Hence, Laser assisted disinfection provided better disinfection and expeditious clinical and radiographic outcome. 
This case series has potential limitations such as shorter duration of follow up, which gives a scope for more research.

\section{Conclusion}

This case series documents the efficacy of laser assisted disinfection with calcium hydroxide intra canal medicament and blood clot as scaffold which showed quantitative outcomes of REP when followed for 12 months with CBCT follow up.

\section{Conflict of Interest}

Declare if any financial interest or any conflict of interest exists.

\section{Bibliography}

1. Lin Jiacheng., et al. "Regenerative Endodontics versus Apexification in Immature Permanent Teeth with Apical Periodontitis: A Prospective Randomized Controlled Study". Journal of Endodontics 43.11 (2017): 1821-1827.

2. Kim Sahng. "Infection and Pulp Regeneration". Dentistry Journal 4.1 (2016): 4.

3. Becerra Patricia., et al. "Histologic Study of a Human Immature Permanent Premolar with Chronic Apical Abscess after Revascularization/Revitalization". Journal of Endodontics 40.1 (2014): 133-139.

4. Vishwanat Lakshmi., et al. "Effect of Bacterial Biofilm on the Osteogenic Differentiation of Stem Cells of Apical Papilla". Journal of Endodontics 43.6 (2017): 916-922.

5. Fouad Ashraf F. "Microbial Factors and Antimicrobial Strategies in Dental Pulp Regeneration". Journal of Endodontics 43.9 (2017).

6. Diogenes Anibal., et al. "An Update on Clinical Regenerative Endodontics". Endodontic Topics 28.1 (2013): 2-23.

7. Kahler Bill and Louis M Lin. "A Review of Regenerative Endodontics: Current Protocols and Future Directions". Journal of Istanbul University Faculty of Dentistry 51 (2017).

8. Hmud Raghad., et al. "Cavitational Effects in Aqueous Endodontic Irrigants Generated by near-Infrared Lasers". Journal of Endodontics 36.2 (2010): 275-278.

9. GUTKNECHT NORBERT., et al. "Diode Laser Radiation and Its Bactericidal Effect in Root Canal Wall Dentin". Journal of Clinical Laser Medicine and Surgery 18.2 (2000): 57-60.
10. El A Ossama R., et al. "Evaluation of the Efficacy of Diode Laser in Maturogenesis of Immature Teeth with Necrotic Pulps: An in Vivo Study "Part One". Indian Journal of Public Health Research and Dentistry 11.03 (2020): 1387.

11. Abdel Hafiz Abdel., et al. "Case Report: Single Visit Photo-Activated Disinfection in Regenerative Endodontics". F1000Research 8 (2020): 1519.

12. Johns Dexton Antony., et al. "Use of Photoactivated Disinfection and Platelet-Rich Fibrin in Regenerative Endodontics". Journal of Conservative Dentistry 17.5 (2014): 487.

13. AAE Clinical Considerations for a Regenerative Procedure Revised 6-8-16, American Association of Endodontics (2016).

14. Naik RashmiG., et al. "Diode Lasers for Pediatric Endodontics: State-of-the-Art!” Journal of Dental Lasers 11.1 (2017): 7.

15. Meschi Nastaran., et al. "A Retrospective Case Series in Regenerative Endodontics: Trend Analysis Based on Clinical Evaluation and 2- and 3-Dimensional Radiology". Journal of Endodontics 44.10 (2018): 1517-1525.

16. Liu C., et al. "Long-Term Exposure to pro-Inflammatory Cytokines Inhibits the Osteogenic/Dentinogenic Differentiation of Stem Cells from the Apical Papilla". International Endodontic Journal 49.10 (2015): 950-959.

17. Lin Louis M., et al. "Histologic and Histobacteriologic Observations of Failed Revascularization/Revitalization Therapy: A Case Report". Journal of Endodontics 40.2 (2014): 291-295.

18. Geisler Todd M. "Clinical Considerations for Regenerative Endodontic Procedures". Dental Clinics of North America 56.3 (2012): 603-626.

19. Kharchi Adam S.., et al. "Regenerative Endodontic Procedures, Disinfectants and Outcomes: A Systematic Review". Primary Dental Journal 9.4 (2020): 65-84.

20. Tunç Hamit., et al. "Evaluation of Low-Level Diode Laser Irradiation and Various IRRIGANT Solutions on the Biological Response of Stem Cells from Exfoliated Deciduous Teeth". Journal of Photochemistry and Photobiology B: Biology 191 (2019): 156-163.

21. Gutknecht N., et al. "Bactericidal Effect of a 980-Nm Diode Laser in the Root Canal Wall Dentin of Bovine Teeth". Journal of Clinical Laser Medicine and Surgery 22.1 (2004): 9-13. 
22. Hmud Raghad., et al. "Cavitational Effects in Aqueous Endodontic Irrigants Generated by near-Infrared Lasers". Journal of Endodontics 36.2 (2010): 275-278.

23. Arslan Hakan., et al. "Effect of Agitation of EDTA with 808-Nanometer Diode Laser on Removal of Smear Layer". Journal of Endodontics 39.12 (2013): 1589-1592.

24. Ruparel Nikita B., et al. "Direct Effect of Intracanal Medicaments on Survival of Stem Cells of the Apical Papilla". Journal of Endodontics 38.10 (2012): 1372-1375.

25. Yilmaz Sehnaz., et al. "The Effect of Antibiotic Pastes on Microhardness of Dentin". Dental Traumatology 32.1 (2015): 27-31.

26. Nagata JY., et al. "Microbial evaluation of traumatized teeth treated with triple antibiotic paste or calcium hydroxide with $2 \%$ chlorhexidine gel in pulp revascularization". Journal of Endodontics 40 (2014): 778-783.

27. Alshehadat Saaid Ayesh., et al. "Scaff Olds for Dental Pulp Tissue Regeneration: A Review". International Dental and Medical Journal of Advanced Research - VOLUME 20152.1 (2016): 1-12.

Volume 5 Issue 12 December 2021

(C) All rights are reserved by Divya Doddamani., et al. 